Check for updates

Cite this: J. Mater. Chem. C, 2021, 9, 1746

Received 10th October 2020, Accepted 20th December 2020 DOI: $10.1039 / \mathrm{d} 0 \mathrm{tc} 04800 \mathrm{c}$

rsc.li/materials-c

\section{Facile synthesis of solid-state fluorescent organosilica nanoparticles with a photoluminescence quantum yield of $73.3 \%$ for fingerprint recognition and white-light-emitting diodes $\dagger$}

\author{
Jiahao Liang, ${ }^{\mathrm{a}}$ Yongzhong $\mathrm{Wu}^{\mathrm{b}}{ }^{\mathrm{X}}$ Xiao Gong (D)*ac and Alberto Vomiero (D)*de
}

\begin{abstract}
Silicon-based fluorescent nanomaterials have attracted widespread attention in latent fingerprint detection and white light-emitting diodes (WLEDs) due to their high photoluminescence, low toxicity and good stability. However, it is still challenging to fabricate solid-state silicon-based nanomaterials with a high quantum yield because of their severe emission quenching properties. Herein, one-step hydrothermal synthesis of polymer-like coated organosilica nanoparticles (OSiNPs) with strong blue luminescence emission by using $N$-[3-(trimethoxysilyl)propyl]ethylenediamine (DAMO), zinc chloride and sodium citrate as precursors is reported. The self-quenching-resistant polymer-like coated OSiNP powder can be easily obtained by ethanol precipitation and oven drying, and the absolute photoluminescence quantum yield (PLQY) can reach up to $73.3 \%$. The possible formation mechanism of polymer-like coated OSiNPs is proposed. Because of the strong solid-state fluorescence, the OSiNP powder can be successfully applied in rapid latent fingerprint detection with enhanced imaging on various substrate surfaces and integrated with commercial phosphor on UV chips to fabricate WLEDs.
\end{abstract}

\section{Introduction}

Silicon-based nanoparticles (SiNPs) have attracted extensive attention due to their special properties such as excellent fluorescence performance, good light stability and high abundance. ${ }^{1-3}$ At the same time, compared with other types of semiconductor QDs and organic dyes, SiNPs have low toxicity and high biocompatibility because of the absence of heavy metal elements, which make them suitable for applications in the fields of solar energy, photoelectrochemistry, photocatalysis,

\footnotetext{
${ }^{a}$ State Key Laboratory of Silicate Materials for Architectures, Wuhan University of Technology, Wuhan 430070, China.

E-mail:xgong@whut.edu.cn

${ }^{b}$ School of Mechanical Engineering, Suzhou University of Science and Technology, Suzhou 215009, China

${ }^{c}$ State Key Laboratory of Polymer Materials Engineering, Sichuan University, Chengdu 610065, China

${ }^{d}$ Division of Materials Science, Department of Engineering Sciences and Mathematics, Luleå University of Technology, 97187 Luleå, Sweden. E-mail: alberto.vomiero@ltu.se

${ }^{e}$ Department of Molecular Sciences and Nano Systems, Ca' Foscari University of Venice, Via Torino 155, 30172 Venezia Mestre, Italy

$\dagger$ Electronic supplementary information (ESI) available. See DOI: 10.1039/ d0tc04800c
}

sensing, and biological imaging. ${ }^{4-10}$ Since Canham discovered the photoluminescence phenomenon of porous silicon at room temperature in $1990,{ }^{11}$ various preparation methods of SiNPs have emerged. ${ }^{12-14}$ So far, most of the studies have focused on adjusting the fluorescence color and photoluminescence quantum yield (PLQY) of SiNPs. ${ }^{15}$ However, the aggregation-induced quenching of the fluorescence of most SiNPs limits their application in many fields such as light emitting diodes, ${ }^{16}$ luminescent solar concentrators ${ }^{17-19}$ and biomarkers (e.g., fingerprint detection $\left.{ }^{20}\right)$. Therefore, it is urgent to develop a method that can inhibit the aggregation-induced quenching of fluorescent SiNPs so that they can maintain their fluorescence properties in solid state for practical use.

Fingerprints with enough complexity are one of the most widely used bases for individual identification. Fingerprints are regarded as significant trace evidence in the judicial systems of various countries. Potential fingerprints are not visible under normal conditions and need to be visualized and identified by special technologies. After a long-term study, widely used fingerprint technologies such as the cyanoacrylate fuming method, the small particle reagent technique and the powdering method have been gradually developed..$^{21}$ Recently, in order to enhance the optical contrast between fingerprints and 
substrates, the application of fluorescent nanomaterials for fingerprint recognition has become the focus of active research. With further development, some inorganic nanoparticles such as gold nanoparticles, ${ }^{22}$ semiconductor quantum dots (QDs) such as CdTe quantum $\operatorname{dots}^{23}$ and magnetic nanoparticles ${ }^{24}$ have been applied for latent fingerprint imaging. However, their complex preparation routes, high toxicity or insufficient PLQY hinder their wide application. Therefore, it is necessary to develop materials with low toxicity through simple fabrication methods with a high PLQY for clear fingerprint visualization. Recently, WLEDs also have attracted extensive attention as long-life and environmentally friendly light sources. ${ }^{25-27}$ WLED lamps are usually excited by ultraviolet chips and mixed with blue, green and red phosphors or excited by blue chips mixed with yellow phosphors. Traditional phosphors for WLEDs are usually made of rare earth materials or Cd-containing semiconductor quantum dot materials. ${ }^{28}$ The shortage of rare earth elements and the toxicity of $\mathrm{Cd}$ limit their applications in WLEDs. Fluorescent SiNP powder with a high PLQY, adjustable fluorescence emission, high thermal and light stability, low cost and environmental friendliness have become new phosphors for WLEDs. At the same time, for lighting applications, the characteristic of narrow emission is often not suitable for obtaining a high color rendering index, which makes it necessary to prepare SiNP powder with a wide emission spectrum for WLED applications.

To solve the aforementioned problems, polymer-like coated OSiNPs with a broad blue emission band are synthesized through a one-step hydrothermal method by using DAMO, zinc chloride and sodium citrate as precursors. The fabrication method is simple and robust. Due to the polymer-like coating, the prepared OSiNPs can maintain strong solid-state fluorescence. The PLQY of blue emission is as high as $73.3 \%$. We demonstrated the successful application of the as-prepared polymer-like coated OSiNPs in rapid latent fingerprint detection and WLEDs.

\section{Experimental section}

\subsection{Materials}

Sodium citrate (98\%), $N$-[3-(trimethoxysilyl)propyl]ethylenediamine (DAMO, 95\%) and zinc chloride $\left(\mathrm{ZnCl}_{2}, \geq 98 \%\right)$ were purchased from Aladdin Chemistry Co., Ltd. Ethanol (EtOH, $\geq 99.7 \%$ ) was purchased from Sinopharm Chemical Reagent Co., Ltd. (Ba, Sr) $)_{2} \mathrm{SiO}_{4}: \mathrm{Eu}^{2+}$ powder, $\mathrm{CaAlSiN}_{3}: \mathrm{Eu}^{2+}$ powder and $365 \mathrm{~nm}$ LED chips were purchased from Looking Long Technology Co., Ltd. Epoxy resins (E51 + W93) were purchased from Kunshan Jiulimei Electronic Materials Co., Ltd.

\subsection{Synthesis of solid-state OSiNPs}

Typically, $1.1587 \mathrm{~g}(4.4 \mathrm{mmol})$ sodium citrate and $0.3 \mathrm{~g}$ (2.2 mmol) zinc chloride were dissolved in $4 \mathrm{~mL}$ of deionized water and stirred at room temperature for $10 \mathrm{~min}$. Then, $1 \mathrm{~mL}$ (4.4 mmol) of DAMO was slowly added into the solution and stirred for a few minutes to obtain a clear solution. Afterwards, the solution was transferred into a $10 \mathrm{~mL}$ Teflon-lined stainless-steel autoclave and kept at $160{ }^{\circ} \mathrm{C}$ for $6 \mathrm{~h}$. After being cooled to room temperature, the solution was purified using a 1000 Da dialysis bag. Then four volumes of EtOH was added into the solution. A large amount of the precipitate can be obtained by simple shaking and mixing. The solid-state OSiNPs were collected by centrifugation at $8000 \mathrm{rpm}$ for $3 \mathrm{~min}$ and finally dried in an oven at $60{ }^{\circ} \mathrm{C}$ for $12 \mathrm{~h}$.

\subsection{Fabrication of the OSiNP-based WLED device}

Typically, OSiNP powder was mixed with commercial (Ba, $\mathrm{Sr})_{2} \mathrm{SiO}_{4}: \mathrm{Eu}^{2+}$ powder with green fluorescence and CaAlSi$\mathrm{N}_{3}: \mathrm{Eu}^{2+}$ powder with red fluorescence. Then the mixed powder and epoxy resin glue A were stirred uniformly and integrated into the $365 \mathrm{~nm}$ LED chips after adding epoxy glue B. Finally, the WLED device can be obtained after drying at $80{ }^{\circ} \mathrm{C}$ for $3 \mathrm{~h}$.

\subsection{Collection and imaging of latent fingerprints}

The powder method was used for the actual detection in latent fingerprint imaging. Firstly, a volunteer was requested to rub his or her fingers on the nose or hair and then to press the finger on various substrate surfaces including glass, weighing paper, aluminized paper, plastic and coin. Subsequently, OSiNP powder was sprinkled on the surface of the latent fingerprints and the excess powder was carefully blown away using a rubber suction bulb. Finally, the fluorescence images were captured using iPhone 11 under $365 \mathrm{~nm}$ UV light irradiation.

\subsection{Characterization}

Ultraviolet-visible (UV-vis) absorption spectra were obtained using an UV-vis spectrophotometer (UV2600, Shimadzu Co., Tokyo, Japan) with a quartz cuvette as the container with a $10 \mathrm{~mm}$ optical path. Photoluminescence (PL) spectroscopy was carried out using a fluorescence spectrophotometer (QM/TM/ NIR, Photon Technology International Co., USA). The PL emission was recorded at different excitation wavelengths in the range of 370-650 $\mathrm{nm}$. The excitation spectrum was recorded by collecting the PL emission at $443 \mathrm{~nm}$. The fluorescence lifetimes were measured using a time-correlated single-photon counting (TCSPC) spectrometer with a $392 \mathrm{~nm}$ NanoLED laser (Horiba DeltaFlex, Jobin Yvon, France). The absolute PLQY of the polymer-like coated OSiNP powder was measured using an UV-NIR quantum yield spectrometer (Quantaurus-QY Plus, Hamamatsu, Japan). Transmission electron microscopy (TEM) was performed using a JEM-2100F electron microscope (JEOL, Japan). The X-ray diffraction (XRD) was performed on a D8 Advance X-ray diffractometer (Bruker, Germany). Fourier transform infrared (FT-IR) spectra were recorded using a FT-IR spectrometer (Nicolet 6700, Thermo Electron Co., USA). The $\mathrm{X}$-ray photoelectron spectra (XPS) were recorded using a photoelectron spectrometer (ESCALAB 250Xi, Thermo Electron Co., USA). The electroluminescence (EL) spectra of WLEDs were measured using an HP9000 LED fast spectrum analyzer. 


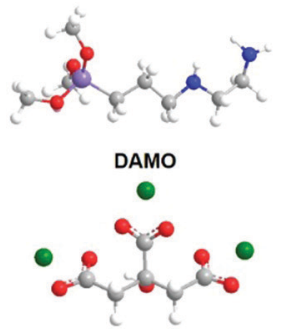

Sodium citrate dihydrate

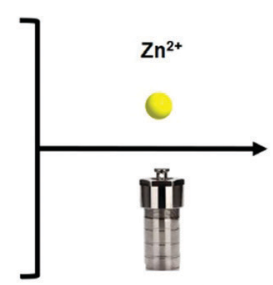

Hydrothermal

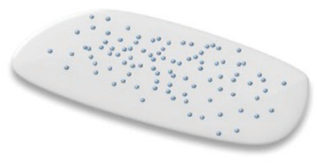

Polymer-like coated OSiNPs

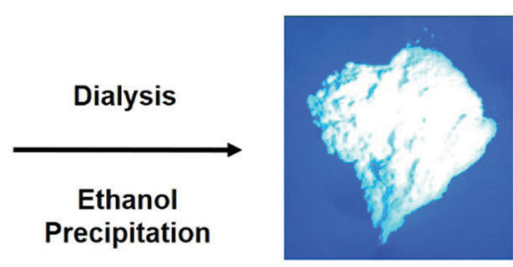

OSiNPs powder

Fig. 1 Schematic illustration of the synthetic route for OSiNP powder.

\section{Results and discussion}

As shown in Fig. 1, the polymer-coated OSiNPs were fabricated via a one-step hydrothermal route using DAMO and sodium citrate as precursors with the assistance of zinc ions. After dialysis, the OSiNP powder was obtained by ethanol precipitation and oven drying.

\subsection{Analysis of the morphology and structure of polymer-like coated OSiNPs}

The TEM image (Fig. 2a) shows that the prepared OSiNPs are wrapped in a polymer-like structure. At a higher magnification (Fig. 2b), it can be observed that the OSiNPs uniformly distribute in the polymer-like structure. In addition, the HRTEM image (Fig. 2b) shows that the polymer-like structure and most of the NPs are amorphous. A few NPs are crystalline with lattice stripes of $\sim 0.19 \mathrm{~nm}$ corresponding to the (220) diffracting plane of silicon, which could be attributed to the breaking of the $\mathrm{Si}-\mathrm{O}$ bond and reduction to silicon under even relatively low intensity electron beams. ${ }^{29-31}$ Some $\mathrm{ZnO}$ crystals are also found in the HRTEM image (Fig. S1, ESI $\dagger$ ), which are most likely the residuals from the synthesis since no signal pertaining to $\mathrm{ZnO}$ is revealed by XRD (Fig. 2d). ${ }^{32}$ According to the statistical calculation of about 100 OSiNPs, the size distribution of OSiNPs is between $2.4 \mathrm{~nm}$ and $6.4 \mathrm{~nm}$ with an average size of $4.4 \mathrm{~nm}$, as shown in Fig. $2 \mathrm{~b}$ (inset).

To identify the structure of polymer-like coated OSiNPs, FT-IR spectroscopy was conducted for the dried OSiNP powder

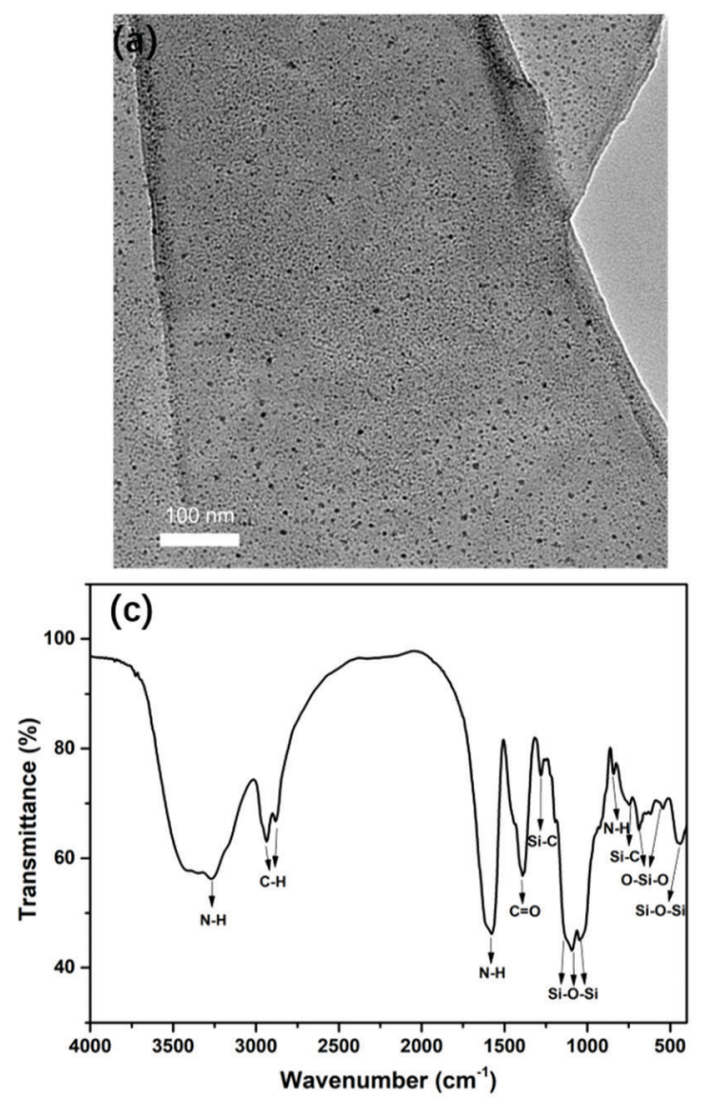

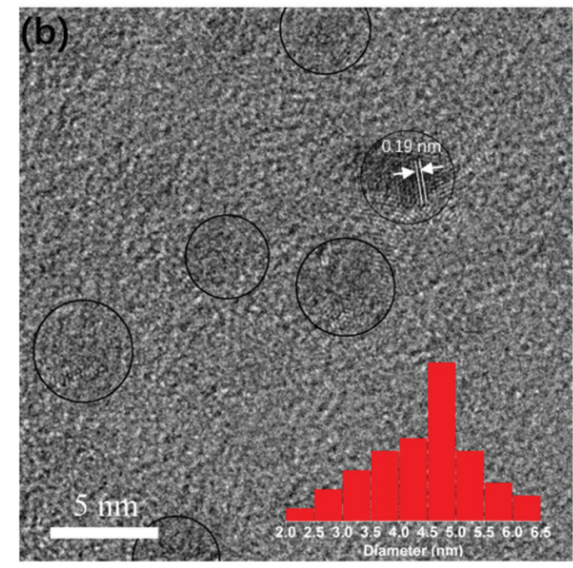

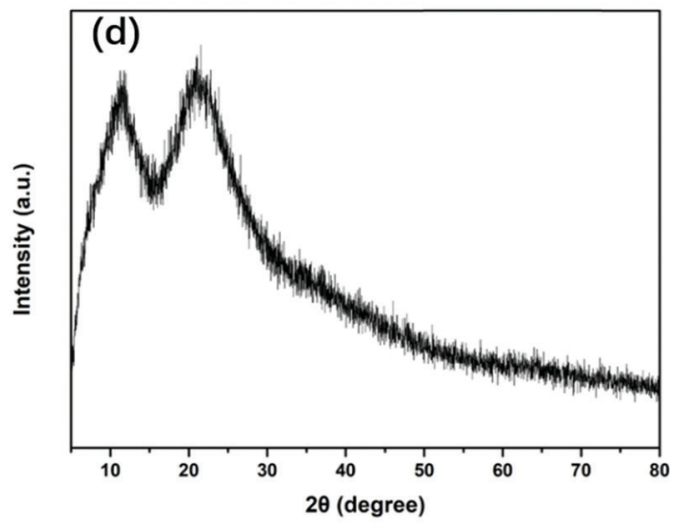

Fig. 2 (a and b) TEM and HRTEM images of polymer-like coated OSiNPs (the inset shows the size distribution of OSiNPs). (c) FTIR spectrum of the OSiNP powder. (d) XRD pattern of the OSiNP powder. 
(Fig. 2c). The FT-IR spectrum shows several distinct absorption peaks in the range of $400-4000 \mathrm{~cm}^{-1}$. The stretching vibrations at $\sim 3269$ and $842 \mathrm{~cm}^{-1}$ and the bending vibration at $\sim 1585 \mathrm{~cm}^{-1}$ are assigned to the $\mathrm{N}-\mathrm{H}$ bond. ${ }^{5,33,34}$ The stretching vibrations of the $\mathrm{C}-\mathrm{H}$ bond occur at $\sim 2936$ and $2882 \mathrm{~cm}^{-1}$. ${ }^{33}$ The absorption at $\sim 1390 \mathrm{~cm}^{-1}$ is assigned to the $\mathrm{C}=\mathrm{O}$ stretching vibration. ${ }^{16}$ The peaks at $\sim 1280 \mathrm{~cm}^{-1}$ and $756 \mathrm{~cm}^{-1}$ are assigned to the stretching and bending vibrations of the $\mathrm{Si}-\mathrm{C}$ bond, respectively. ${ }^{35-37}$ The strong absorption peaks at $\sim 1133,1090$ and $1033 \mathrm{~cm}^{-1}$ are ascribed to the stretching vibration of $\mathrm{Si}-\mathrm{O}-\mathrm{Si}$. The peaks located at $\sim 1133$ and $1090 \mathrm{~cm}^{-1}$ belong to the $\mathrm{Si}-\mathrm{O}-\mathrm{Si}$ cage and the ring/network structure, respectively, and the peak at $1033 \mathrm{~cm}^{-1}$ can be attributed to the $\mathrm{Si}-\mathrm{O}-\mathrm{Si}$ chain structure. ${ }^{38-40}$ The absorption at $440 \mathrm{~cm}^{-1}$ belongs to the bending vibration of $\mathrm{Si}-\mathrm{O}-\mathrm{Si}^{36,37,41}$ The peaks at 688 and $544 \mathrm{~cm}^{-1}$ are due to the stretching and bending vibrations of $\mathrm{O}-\mathrm{Si}-\mathrm{O} .{ }^{36,42}$ In the XRD pattern (Fig. 2d), the polymer-like coated OSiNPs display two broad peaks. The peak at $\sim 22^{\circ}$ is attributed to the amorphous silica and the peak at $\sim 11^{\circ}$ can be attributed to the silicone networks. ${ }^{43-45}$ Because the OSiNPs are uniformly distributed in the polymer-like film, the phenomenon of aggregation quenching can be well avoided, which may be an important factor in achieving the high solid-state fluorescence of OSiNPs.

In addition, XPS measurements were performed to further analyze the chemical composition of the polymer-like coated OSiNP structure. As shown in Fig. 3a, the full-scan XPS spectrum of the OSiNP powder distinctly reveals the presence of $\mathrm{C}$ 1s, N 1s, O 1s, Na 1s, Zn 2p, Zn 3p, Si 2p and Cl 2p. In the highresolution analysis, the $\mathrm{C} 1 \mathrm{~s}$ can be fitted into five peaks at $283.5 \mathrm{eV}, 284.6 \mathrm{eV}, 285.4 \mathrm{eV}, 286.4 \mathrm{eV}$ and $288 \mathrm{eV}$, which are attributed to $\mathrm{C}-\mathrm{Si}, \mathrm{C}-\mathrm{C}, \mathrm{C}-\mathrm{N}, \mathrm{C}-\mathrm{O}$ and $\mathrm{C}=\mathrm{O}$, respectively, ${ }^{46-48}$ as shown in Fig. $3 \mathrm{~b}$. The $\mathrm{N} 1 \mathrm{~s}$ band can be deconvoluted into two peaks at $399 \mathrm{eV}$ and $400.4 \mathrm{eV}$ (Fig. 3c), which are assigned to the $\mathrm{C}-\mathrm{N}-\mathrm{C}$ and $\mathrm{C}-\mathrm{NH}_{2}$, respectively. ${ }^{5,6}$ The $\mathrm{O} 1 \mathrm{~s}$ band contains three peaks at $531.0 \mathrm{eV}, 532.0 \mathrm{eV}$ and $535.5 \mathrm{eV}$ (Fig. 3d), which are attributed to the $\mathrm{C}-\mathrm{O}, \mathrm{Si}-\mathrm{O}$ and adsorbed free oxygen, respectively. ${ }^{37,49,50}$ The Si $2 \mathrm{p}$ band can be resolved into two peaks at 101.0 and $101.8 \mathrm{eV}$ (Fig. 3e), corresponding to $\mathrm{Si}-\mathrm{C}$ and Si-O, respectively. ${ }^{51-53}$ The $\mathrm{Zn} 2 \mathrm{p}$ band can be split into $\mathrm{Zn}$ $2 \mathrm{p}_{3 / 2}$ at $1021.5 \mathrm{eV}$ and $\mathrm{Zn} 2 \mathrm{p}_{1 / 2}$ at $1044.7 \mathrm{eV}$, as shown in Fig. 3f, indicating the existence of $\mathrm{Zn}$ element with a +2 valence state. ${ }^{54}$

\subsection{Fluorescence properties of polymer-like coated OSiNPs}

The UV-vis spectrum of polymer-like coated OSiNPs dispersed in aqueous solution shows a broad absorption ranging from 300 to $420 \mathrm{~nm}$ with the maximum absorption peak at $\sim 362 \mathrm{~nm}$, which could be attributed to the organic ligand on the OSiNPs, as shown in Fig. 4a. The photographs of OSiNPs dispersed in aqueous solution under daylight and an UV lamp with $365 \mathrm{~nm}$ emission are presented in the inset of Fig. 4a. As the excitation wavelength changes from 200 to $420 \mathrm{~nm}$, the PL intensity increases first and then decreases, with the strongest PL emission intensity excited at $369 \mathrm{~nm}$ (dotted line in Fig. 4b). The polymer-like coated OSiNPs dispersed in aqueous solution show strong blue luminescence emission under $365 \mathrm{~nm}$ excitation and present an emission peak at $\lambda_{\mathrm{em}}=443 \mathrm{~nm}$, which is independent of the excitation wavelength, when excited with wavelengths in the range from $350 \mathrm{~nm}$ to $395 \mathrm{~nm}$ (colored solid lines in Fig. 4b). The transient PL intensity presents a decay compatible with a single exponential decay curve. The fluorescence lifetime of polymer-like coated OSiNPs dispersed in aqueous solution is 14.3 ns (Fig. 4c).
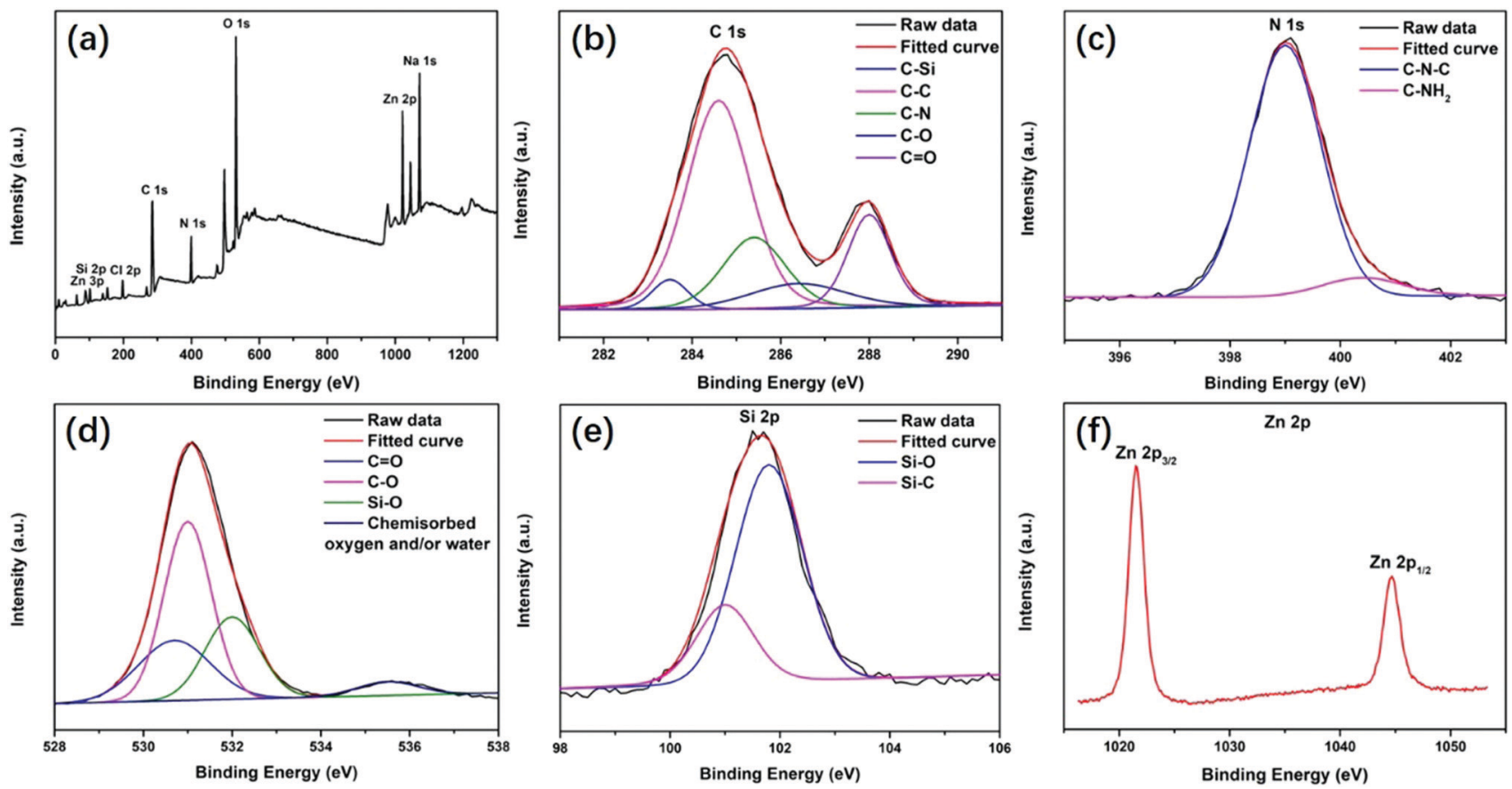

Fig. 3 XPS spectra of the OSiNP powder: (a) full-scan spectrum of the OSiNP powder and high-resolution XPS peaks of (b) C 1s, (c) N 1s, (d) O 1s, (e) Si 2p and (f) Zn 2p. 

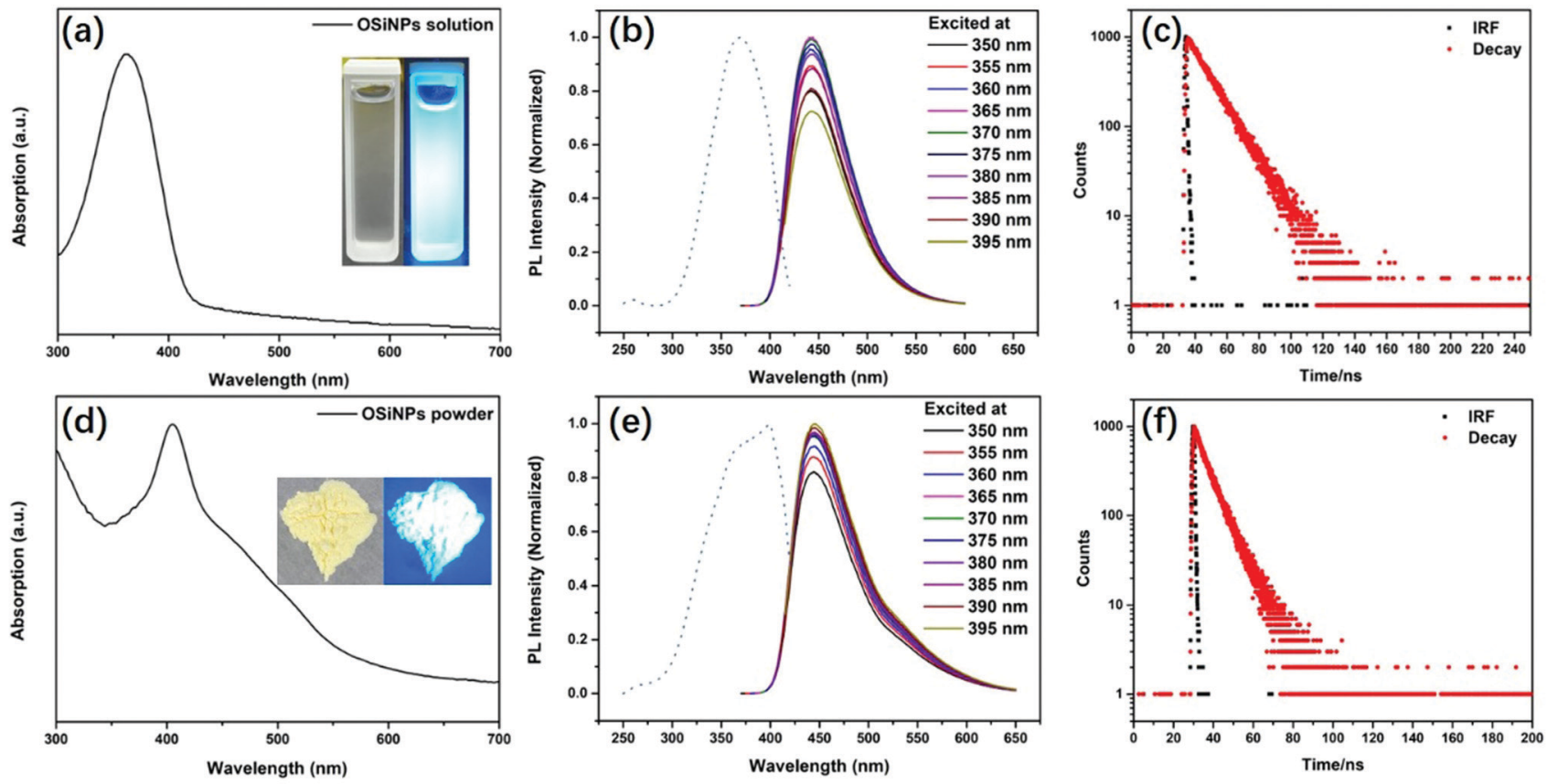

Fig. 4 (a) UV-vis spectra of polymer-like coated OSiNPs dispersed in aqueous solution (inset: the photos of polymer-like coated OSiNPs dispersed in aqueous solution under daylight (left) and $365 \mathrm{~nm}$ UV light (right)). (b) PL excitation (dotted line, $\lambda_{\mathrm{em}}=443 \mathrm{~nm}$ ) and excitation-independent (solid line) spectra of polymer-like coated OSiNPs dispersed in aqueous solution. (c) Fluorescence decay curves of polymer-like coated OSiNPs dispersed in aqueous solution. Red dots: experimental data. Black dots: $392 \mathrm{~nm}$ lased pulse. (d) UV-vis spectra of the polymer-like coated OSiNP powder (inset: the photos of the polymer-like coated OSiNP powder under daylight (left) and $365 \mathrm{~nm}$ UV light (right)). (e) PL excitation (dotted line, $\lambda_{\mathrm{em}}=443 \mathrm{~nm}$ ) and excitation-independent (solid line) spectra of the polymer-like coated OSiNP powder. (f) Fluorescence decay curves of the polymer-like coated OSiNP powder. Red dots: experimental data. Black dots: $392 \mathrm{~nm}$ lased pulse.

Unlike the self-quenching carbon dot powder, the polymerlike coated OSiNPs powder shows strong blue fluorescence under $365 \mathrm{~nm}$ UV light, as shown in the inset of Fig. $4 \mathrm{~d} . .^{55} \mathrm{In}$ addition, the OSiNP powder presents a strong absorption peak at $404 \mathrm{~nm}$. The qualitative trend of PL for the OSiNP powder under excitation in the $350-395 \mathrm{~nm}$ range is similar to that for the polymer-like coated OSiNPs dispersed in aqueous solution. When the excitation wavelength increases, the PL intensity of the polymer-like coated OSiNP powder increases first and then decreases, with the maximum PL intensity observed under excitation at $398 \mathrm{~nm}$ (dotted line in Fig. $4 \mathrm{e}, \lambda_{\mathrm{em}}=444 \mathrm{~nm}$ ). The maximum emission intensity is recorded at $444 \mathrm{~nm}$ with a broad full width at half maximum (FWHM) of $\sim 75 \mathrm{~nm}$, which is almost the same as that for the polymer-like coated OSiNPs dispersed in aqueous solution, as reported in Fig. 4e. By the adjustment of the amount of sodium citrate, the absolute PLQY of the polymer-like coated OSiNP powder can reach $73.3 \%$ under $365 \mathrm{~nm}$ excitation. The PL intensity presents a single exponential decrease. The fluorescence lifetime of the polymerlike coated OSiNP powder is 7.6 ns (Fig. 4f), which is shorter than that of the aqueous solution, indicating that the resonance energy transfer (RET) occurs in the polymer-like coated OSiNP powder. ${ }^{48}$

\subsection{Study of the structure of polymer-like coated OSiNPs}

In order to understand the formation of the polymer-like coated OSiNP structure, we have prepared the sample without $\mathrm{Zn}^{2+}$ under the same conditions. TEM (Fig. S2, ESI $\dagger$ ) shows that the
OSiNPs synthesized without $\mathrm{Zn}^{2+}$ are not coated by the polymerlike structure. Unlike the polymer-like coated OSiNPs, the XRD pattern of OSiNPs synthesized without $\mathrm{Zn}^{2+}$ (Fig. S3, ESI $\dagger$ ) displays no peak at $\sim 11^{\circ}$ and shows only one broad peak at $\sim 22^{\circ}$, which indicates the absence of the silicone network structure. Furthermore, the PL excitation and excitationindependent spectra show that the OSiNPs synthesized without $\mathrm{Zn}^{2+}$ have blue luminescence emission under $365 \mathrm{~nm}$ excitation and present an emission peak at $\lambda_{\mathrm{em}}=444 \mathrm{~nm}$, as shown in Fig. S4 (ESI $\dagger$ ), which is almost the same as that of the polymer-like coated OSiNPs dispersed in aqueous solution, indicating that the polymer-like structure has a little influence on the fluorescence performance. Therefore, $\mathrm{Zn}^{2+}$ affects the formation of the polymer-like structure but has almost no influence on the formation and emission properties of OSiNPs. To further recognize the effect of $\mathrm{Zn}^{2+}$ and the structure of OSiNPs, the FT-IR spectra of OSiNPs synthesized with different amounts of $\mathrm{Zn}^{2+}$ are obtained. The FT-IR spectra (Fig. S5, ESI $\dagger$ ) show that the vibration of $\mathrm{Si}-\mathrm{O}-\mathrm{Si}$ at $1090 \mathrm{~cm}^{-1}$ increases and the vibration of Si-OH at $918 \mathrm{~cm}^{-1}$ decreases with the increase of the $\mathrm{Zn}^{2+}$ content (from 0 to $2.2 \mathrm{mmol}$ ), indicating that $\mathrm{Zn}^{2+}$ promotes the formation of the $\mathrm{Si}-\mathrm{O}-\mathrm{Si}$ ring/network structure, which could be attributed to the polymer-like structure. Apart from the two vibration peaks, the other peaks in these samples are approximately the same. The absorption at $\sim 1133$ and $1033 \mathrm{~cm}^{-1}$ appears in OSiNPs synthesized both with and without $\mathrm{Zn}^{2+}$, indicating that the Si-O-Si cage and chain structures could exist in OSiNPs. ${ }^{38-40}$ Through the above analysis, the NPs 


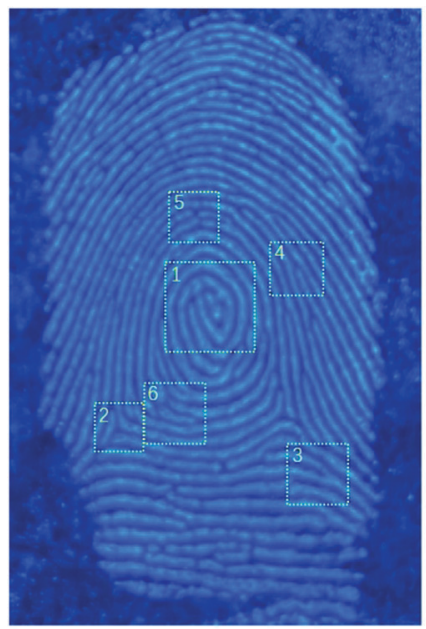

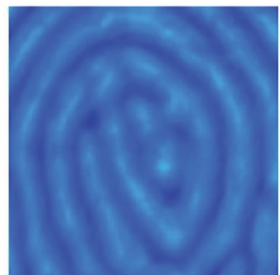

1. Whorl

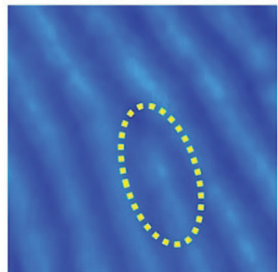

4. Termination

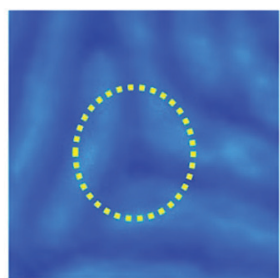

2. Delta

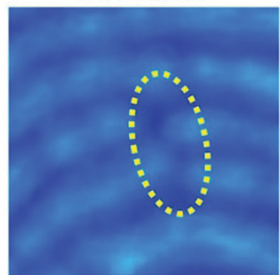

5. Scar

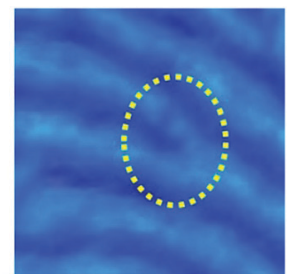

3. Bifurcation

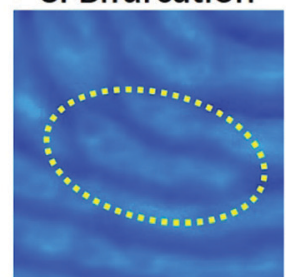

6. Island

Fig. 5 Representative fluorescence image (left panel) and magnified features (right panel) of latent fingerprint development on the aluminum foil with the polymer-like coated OSiNP powder.

are mainly composed of $\mathrm{Si}-\mathrm{O}-\mathrm{Si}$ cage and chain structures with covalently linked fluorescent centers. The rigid structure could weaken the intramolecular vibration and reduce the nonradiative transition of electrons, resulting in the high fluorescence. The polymer-like structure can be composed of silicone networks with the Si-O-Si ring/network structure, which could prevent the damage of the florescent group during aggregation and maintain a high PLQY in solid state.

\subsection{Application of the polymer-like coated OSiNP powder in fingerprint detection}

Fingerprints, as human identification cards, have a very low repetition rate. That is to say, fingerprints with different shapes and ridge widths vary from person to person. The fingerprints have various shapes such as arched, ring-shaped, and threaded with a ridge width of several hundred micrometers, which is much larger than that of the nanoscale polymer-like coated OSiNPs. ${ }^{56}$ Due to the small size, strong fluorescence and simple preparation, the polymer-like coated OSiNP powder holds great potential in latent fingerprint detection. The mechanism of fingerprint detection is the same as that of conventional powder detection, that is, the functional groups such as amide, amine and carboxylic can interact with basic proteins or oily substances. ${ }^{20}$ In practical applications, fingerprint identification is usually divided into three levels. ${ }^{57}$ Patterns or bulges are the first-level features. The second level contains more

\section{Daylight}
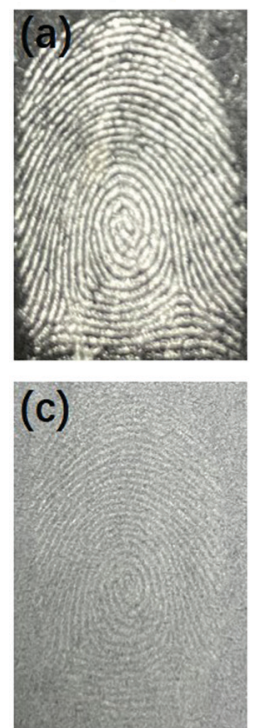

UV light
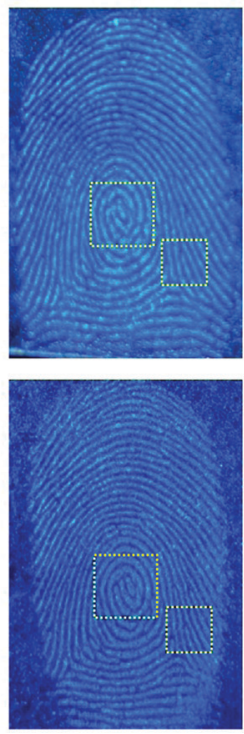
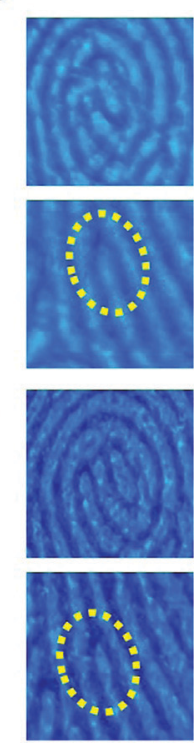

\section{Daylight}
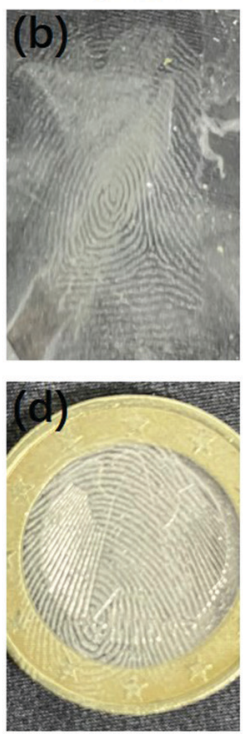

UV light
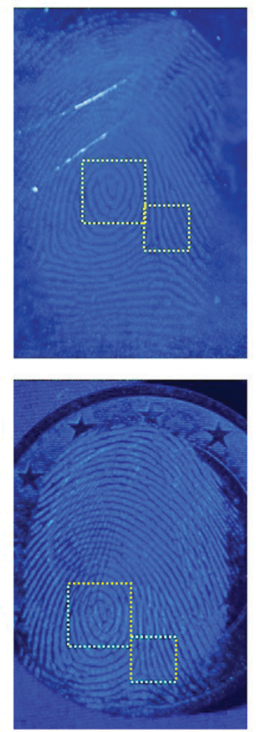
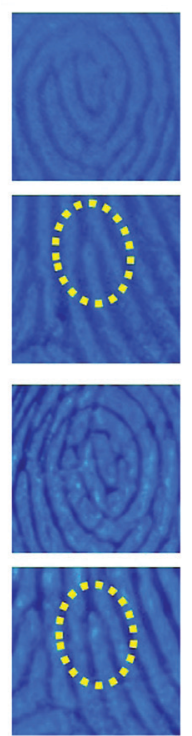

Fig. 6 Images of latent fingerprint development on (a) glass, (b) plastic, (c) weighing paper and (d) a coin with polymer-coated OSiNP powder under daylight (left panel) and UV light (middle and right panels). Details from the main panels from UV irradiation are collected in the right panels, collecting whorl and termination of the fingerprint. 

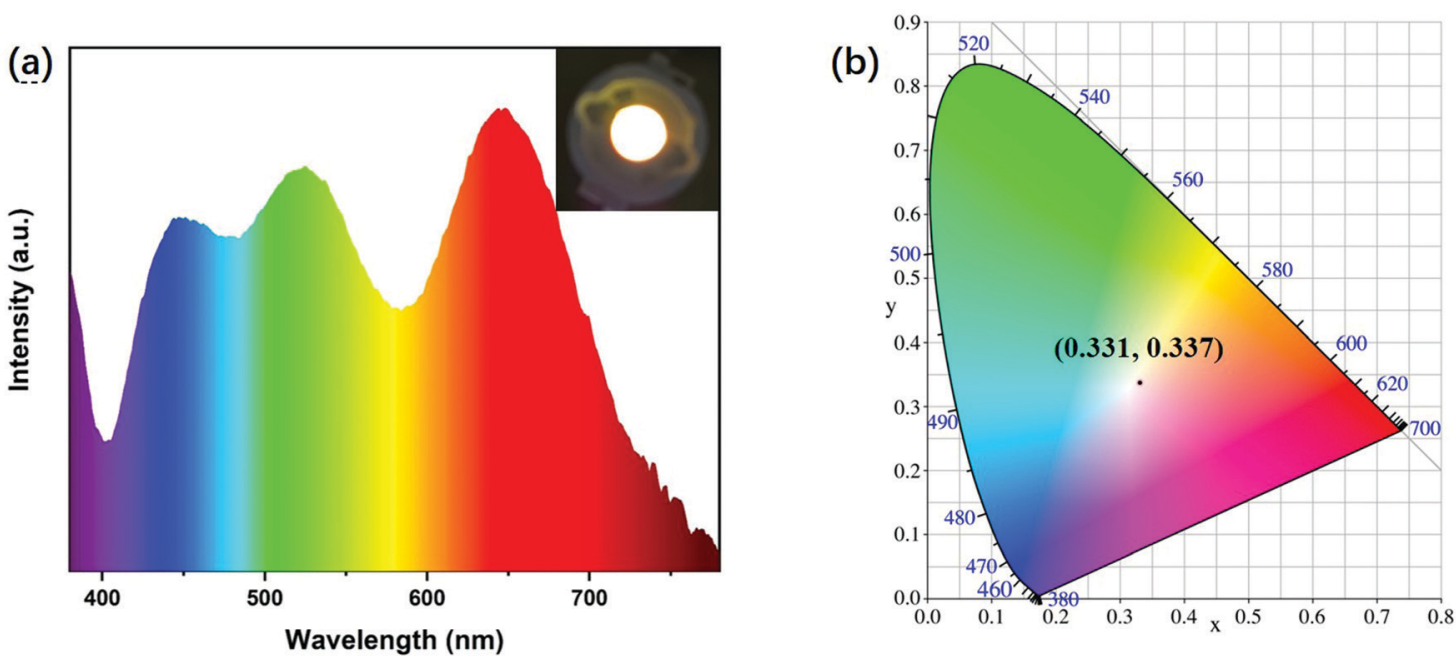

Fig. 7 (a) PL emission spectra of the OSiNP-based WLED (inset: fluorescence images of the WLED). (b) CIR chromaticity coordinates of the WLED.

prominent details of the fingerprint such as bifurcation and termination and the size characteristics of the ridge are considered as the third level details including holes, path deviations of the ridge, and scars. The second and third levels of fingerprints accurately and quantitatively provide fingerprint identification data. ${ }^{56}$ By using the as-prepared fluorescent powder on the finger-printed aluminum foil, the three-level features of texture of the fingerprint such as whorl, delta, bifurcation, termination, scar and island can be clearly observed, as shown in Fig. 5.

As shown in Fig. 6, the color of fingerprint fluorescence appearance on different surfaces does not change, indicating that the PL properties of the polymer-like coated OSiNP powder are stable and not affected by the chemical properties of different substrate surfaces. In addition, the displayed patterns or ridges can be distinctly distinguished and the details of the fingerprint such as bifurcation and whorl can be clearly seen. Because of the achievement of the fingerprint imaging on different substrates with very high resolution and contrast in ridge and space, the prepared polymer-like coated OSiNP powder can realize fingerprint visualization on different substrates without post-processing.

\subsection{Application of the polymer-like coated OSiNP powder in WLEDs}

Due to their high solid-state PLQY, a broad FWHM and no excitation-dependent properties, the polymer-like coated OSiNPs can be used for WLED devices. In this work, we mixed the powder with the commercial green silicate powder $\left((\mathrm{Ba}, \mathrm{Sr})_{2-}\right.$ $\left.\mathrm{SiO}_{4}: \mathrm{Eu}^{2+}\right)$ and red nitride powder $\left(\mathrm{CaAlSiN}_{3}: \mathrm{Eu}^{2+}\right)$. The WLED has been successfully prepared by depositing the mixture on a $365 \mathrm{~nm}$ ultraviolet chip with epoxy resin. The fluorescence image of the WLED and the emission spectrum at a driving current of $20 \mathrm{~mA}$ are shown in Fig. 7a. The color coordinates of the WLED are located at $(0.331,0.337)$, as shown in Fig. $7 \mathrm{~b}$, which are comparable to those of pure white light $(0.33,0.33)$. In addition, the WLED has a color rendering index (CRI) of 79.8 and the color temperature (CCT) is $5568 \mathrm{~K}$. As a result, the polymer-like coated OSiNP powder can be used as a substitute for the commercial fluorescent blue powder to meet the requirements of WLED displays.

\section{Conclusions}

In conclusion, polymer-like coated OSiNPs with strong blueemitting solid-state fluorescence were prepared by a one-step hydrothermal method with the assistance of $\mathrm{Zn}^{2+}$. The luminescent OSiNPs are confined in the polymer-like matrix uniformly, which can effectively prevent them from aggregation-induced luminescence quenching. The prepared fluorescent powder had a high PLQY of $73.3 \%$ and was successfully applied to fingerprint recognition on different substrates. The displayed fingerprint image had a high contrast with the background and the three-level details of the fingerprint were clearly visible. Meanwhile, due to the solid-state fluorescence properties of polymer-like coated OSiNPs, they can be employed for fabricating WLEDs deposited with commercial green and red fluorescent powders on $365 \mathrm{~nm}$ chips. In addition, because of its low toxicity, easy preparation and strong solid-state fluorescence, the polymer-like coated OSiNP powder has great potential in photoelectric devices and biomarkers.

\section{Conflicts of interest}

There are no conflicts to declare.

\section{Acknowledgements}

This work was supported by the National Natural Science Foundation of China (No. 21774098) and the Open Foundation of the State Key Laboratory of Silicate Materials for Architectures at WUT (No. SYSJJ2018-04). This study was also financially supported by the Opening Project of the State Key Laboratory of Polymer Materials Engineering (Sichuan University) (Grant No. sklpme2019-4-26). A. V. acknowledges 
the Kempe Foundation and the Knut and Alice Wallenberg Foundation for financial support.

\section{References}

1 F. Peng, Y. Su, Y. Zhong, C. Fan, S.-T. Lee and Y. He, Acc. Chem. Res., 2014, 47, 612-623.

2 J. Liang, C. Huang and X. Gong, ACS Sustainable Chem. Eng., 2019, 7, 18213-18227.

3 M. Dasog, J. Kehrle, B. Rieger and J. G. C. Veinot, Angew. Chem., Int. Ed., 2016, 55, 2322-2339.

4 S. Abdulhussain, H. Breitzke, T. Ratajczyk, A. Grunberg, M. Srour, D. Arnaut, H. Weidler, U. Kunz, H. J. Kleebe, U. Bommerich, J. Bernarding, T. Gutmann and G. Buntkowsky, Chem. - Eur. J., 2014, 20, 1159-1166.

5 Y. X. Han, Y. L. Chen, J. Feng, J. J. Liu, S. D. Ma and X. G. Chen, Anal. Chem., 2017, 89, 3001-3008.

6 X. Chen, X. Zhang, L. Y. Xia, H. Y. Wang, Z. Chen and F. G. Wu, Nano Lett., 2018, 18, 1159-1167.

7 Y.-K. Dou, Y. Chen, X.-W. He, W.-Y. Li, Y.-H. Li and Y.-K. Zhang, Anal. Chem., 2017, 89, 11286-11292.

8 R. Mazzaro, A. Gradone, S. Angelon, G. Morselli, P. G. Cozzi, F. Romano, A. Vomiero and P. Ceroni, ACS Photonics, 2019, 6, 2303-2311.

9 K. Yorseng, S. Siengchin, B. Ashok and A. V. Rajulu, J. Bioresour. Bioprod., 2020, 5, 101-107.

10 B. Ashok, N. Hariram, S. Siengchin and A. V. Rajulu, J. Bioresour. Bioprod., 2020, 5, 180-185.

11 L. T. Canham, Appl. Phys. Lett., 1990, 57, 1046-1048.

12 S. Guruvenket, J. M. Hoey, K. J. Anderson, M. T. Frohlich, R. Krishnan, J. Sivaguru, M. P. Sibi and P. Boudjouk, J. Mater. Chem. C, 2016, 4, 8206-8213.

13 H. L. Ye, S. J. Cai, S. Li, X. W. He, W. Y. Li, Y. H. Li and Y. K. Zhang, Anal. Chem., 2016, 88, 11631-11638.

14 X. K. Chen, X. D. Zhang, C. C. Li, S. M. Sayed, W. Sun, F. M. Lin and F. G. Wu, Sens. Actuators, B, 2019, 295, 49-55.

15 S. Chandra, B. Ghosh, G. Beaune, U. Nagarajan, T. Yasui, J. Nakamura, T. Tsuruoka, Y. Baba, N. Shirahata and F. M. Winnik, Nanoscale, 2016, 8, 9009-9019.

16 C.-L. Shen, J.-H. Zang, Q. Lou, L.-X. Su, Z. Li, Z.-Y. Liu, L. Dong and C.-X. Shan, Carbon, 2018, 136, 359-368.

17 Z. Wang, X. Zhao, Z. Guo, P. Miao and X. Gong, Org. Electron., 2018, 62, 284-289.

18 W. Ma, W. Li, M. Cao, R. Liu, X. Zhao and X. Gong, Org. Electron., 2019, 73, 226-230.

19 X. Gong, W. Ma, Y. Li, L. Zhong, W. Li and X. Zhao, Org. Electron., 2018, 63, 237-243.

20 H.-J. Wang, W.-Y. Hou, T.-T. Yu, H.-L. Chen and Q.-Q. Zhang, Dyes Pigm., 2019, 170, 107623.

21 S. Hong, J.-H. Park, J.-H. Park, H.-b. Oh, E.-J. Choi, I.-H. Cho and Y.-J. Mok, J. Forensic Sci., 2019, 64, 1040-1047.

22 H.-W. Tang, W. Lu, C.-M. Che and K.-M. Ng, Anal. Chem., 2010, 82, 1589-1593.

23 J. Liu, Z. Shi, Y. Yu, R. Yang and S. Zuo, J. Colloid Interface Sci., 2010, 342, 278-282.
24 O. S. Wolfbeis, Angew. Chem., Int. Ed., 2009, 48, 2268-2269. 25 R. Cao, Y. Cao, T. Fu, S. Jiang, W. Li, Z. Luo and J. Fu, J. Alloys Compd., 2016, 661, 77-81.

26 L. Li, X. Tang, Z. Jiang, X. Zhou, S. Jiang, X. Luo, G. Xiang and K. Zhou, J. Alloys Compd., 2017, 701, 515-523.

27 R. Cao, C. Liao, F. Xiao, G. Zheng, W. Hu, Y. Guo and Y. Ye, Dyes Pigm., 2018, 149, 574-580.

28 B. S. Mashford, M. Stevenson, Z. Popovic, C. Hamilton, Z. Zhou, C. Breen, J. Steckel, V. Bulovic, M. Bawendi, S. Coe-Sullivan and P. T. Kazlas, Nat. Photonics, 2013, 7, 407-412.

29 J. R. Pitts and A. W. Czanderna, Nuclear Instruments and Methods in Physics Research Section B: Beam Interactions with Materials and Atoms, 1986, vol. 13, pp. 245-249.

30 P. Buckley, N. Hargreaves and S. Cooper, Commun. Chem., 2018, 1, 49.

31 J. L. Z. Ddungu, S. Silvestrini, A. Tassoni and L. De Cola, Faraday Discuss., 2020, 222, 350-361.

32 Y. Lai, M. Meng and Y. Yu, Appl. Catal., B, 2010, 100, 491-501.

33 F. G. Wu, X. D. Zhang, S. Q. Kai, M. Y. Zhang, H. Y. Wang, J. N. Myers, Y. X. Weng, P. D. Liu, N. Gu and Z. Chen, Adv. Mater. Interfaces, 2015, 2, 1500360.

34 F. Chen, T. Zhao, Q. Chen, L. Han, S. Fang and Z. Chen, Mater. Lett., 2013, 108, 179-182.

35 P. K. Sudeep, Z. Page and T. Emrick, Chem. Commun., 2008, 6126, DOI: 10.1039/b813025f.

36 E. S. Park, H. W. Ro, C. V. Nguyen, R. L. Jaffe and D. Y. Yoon, Chem. Mater., 2008, 20, 1548-1554.

37 A. Guleria, A. P. Chavan, S. Neogy, V. V. Gandhi, A. Kunwar, A. K. Debnath and S. Adhikari, ACS Appl. Nano Mater., 2020, 3, 5123-5138.

38 C.-C. Yang and W.-C. Chen, J. Mater. Chem., 2002, 12, 1138-1141.

39 S. Liu, X. Lang, H. Ye, S. Zhang and J. Zhao, Eur. Polym. J., 2005, 41, 996-1001.

40 H. Nagasawa, T. Minamizawa, M. Kanezashi, T. Yoshioka and T. Tsuru, J. Membr. Sci., 2015, 489, 11-19.

41 L. Yang, L. Wang, C. Cui, J. Lei and J. Zhang, Chem. Commun., 2016, 52, 6154-6157.

42 D. Xing, L. Chen, Q. Ma, B. Hao, S. I. Gutnikov, B. I. Lazoryak, E. Mäder and P.-C. Ma, J. Non-Cryst. Solids, 2020, 548, 120331.

43 W. Wang, R. Liu, W. Liu, J. Tan, W. Liu, H. Kang and Y. Huang, J. Mater. Sci., 2010, 45, 5567-5573.

44 L. Liu, M. Tian, W. Zhang, L. Zhang and J. E. Mark, Polymer, 2007, 48, 3201-3212.

45 L. Li, R. Liang, Y. Li, H. Liu and S. Feng, J. Colloid Interface Sci., 2013, 406, 30-36.

46 F. Li, H. Li and T. Cui, Opt. Mater., 2017, 73, 459-465.

47 Y. Zhan, B. Shang, M. Chen and L. Wu, Small, 2019, 15, 1901161.

48 T. Gong, Y. Li, B. Lei, X. Zhang, Y. Liu and H. Zhang, J. Mater. Chem. C, 2019, 7, 5962-5969.

49 Y. Liu, Q. Zhou, Y. Yuan and Y. Wu, Carbon, 2017, 115, 550-560. 
50 A. Olejniczak, M. Lezanska, J. Wloch, A. Kucinska and J. P. Lukaszewicz, J. Mater. Chem. A, 2013, 1, 8961-8967.

51 Y.-S. Wei, W.-Y. Liu, H.-M. Wu, K.-S. Chen and V. Cech, Mater. Chem. Phys., 2017, 189, 183-190.

52 Q. Wang, Y. Kawano, L. Yu, H. Nagasawa, M. Kanezashi and T. Tsuru, J. Membr. Sci., 2020, 598, 117688.

53 M. Li, L. Jiang, Y. Sun, T. Xiao, P. Xiang and X. Tan, J. Alloys Compd., 2018, 753, 320-328.
54 M. Zhu, Y. Li, S. Tian, Y. Xie, X. Zhao and X. Gong, J. Colloid Interface Sci., 2019, 534, 509-517.

55 M. Sun, S. Qu, Z. Hao, W. Ji, P. Jing, H. Zhang, L. Zhang, J. Zhao and D. Shen, Nanoscale, 2014, 6, 13076-13081.

56 H. Zhang, J. You, C. Nie, J. Wang, X. Dong, R. Guan, D. Cao and Q. Chen, J. Lumin., 2019, 215, 116582.

57 Y.-H. Chen, S.-Y. Kuo, W.-K. Tsai, C.-S. Ke, C.-H. Liao, C.-P. Chen, Y.-T. Wang, H.-W. Chen and Y.-H. Chan, Anal. Chem., 2016, 88, 11616-11623. 